\title{
A NOTE ON INTERTWINING $M$-HYPONORMAL OPERATORS
}

\author{
R. L. MOORE, D. D. ROGERS AND T. T. TRENT ${ }^{1}$
}

\begin{abstract}
ABSTRACr. If $A X=X B^{*}$ with $A$ and $B M$-hyponormal, then $A^{*} X=X B$. Furthermore, $(\operatorname{ran} X)^{-}$reduces $A$, ker $X$ reduces $B$, and $A \mid(\operatorname{ran} X)^{-}$and $B^{*} \mid k^{\perp}{ }^{\perp} X$ are unitarily equivalent normal operators. An asymptotic version is also proved.
\end{abstract}

Let $\mathcal{H}$ be a Hilbert space. A bounded operator $A$ on $\mathcal{H}$ is called dominant by $\mathrm{J}$. Stampfli and B. Wadhwa [4] if, for all complex $\lambda, \operatorname{range}(A-\lambda) \subseteq \operatorname{range}(A-\lambda)^{*}$, or, equivalently, if there is a real number $M_{\lambda} \geqslant 1$ such that

$$
\left\|(A-\lambda)^{*} f\right\| \leqslant M_{\lambda}\|(A-\lambda) f\|
$$

for all $f$ in $\mathcal{H}$. If there is a constant $M$ such that $M_{\lambda}<M$ for all $\lambda, A$ is called $M$-hyponormal, and if $M=1, A$ is hyponormal.

Stampfli and Wadhwa showed in [4, Theorem 1] that if $A$ is dominant, $B$ is hyponormal, $X$ is one-to-one and has dense range, and if $A X=X B^{*}$, then $A$ and $B$ are normal. M. Radjabalipour improved this result by allowing $B$ to be $M$-hyponormal [3, Theorem 3(a)]. Of course, the condition that $A$ and $B$ are normal allows one to conclude immediately by the usual Putnam-Fuglede theorem that $A^{*} X=$ $X B$. S. K. Berberian [2] has obtained the latter result under the conditions that $A$ and $B$ are hyponormal and $X$ is Hilbert-Schmidt (but not one-to-one or with dense range). It seems to have escaped notice, however, that if $A$ and $B$ are both $M$-hyponormal, the conclusion that $A^{*} X=X B$ can be reached with no restrictions on $X$ at all; moreover, by employing both intertwining equations one can determine precisely the subspaces on which $A$ and $B$ must be normal. We will need two other results from [3]:

Theorem A (RAdjabalipour). Let $A$ be dominant and let $\mathfrak{T}$ be an invariant subspace of $A$ for which $A \mid \Re$ is normal. Then $\Re$ reduces $A$.

TheOREM B (RADJABalipour). If $A$ and $A^{*}$ are $M$-hyponormal then $A$ is normal.

We begin with a symmetric version.

Theorem 1. Let $A$ be $M$-hyponormal and suppose that $A X=X A^{*}$. Then $A^{*} X=$ $X A$.

Received by the editors September 12, 1980.

1980 Mathematics Subject Classification. Primary 47B20.

Key words and phrases. Normal operator, $M$-hyponormal operator, intertwining.

${ }^{1}$ The first and third authors were partially supported by grants from the Research Grants Committee of the University of Alabama. 
Proof. Let $X=H+i J$ be the Cartesian decomposition of $X$. By taking the adjoint of the intertwining equation, we obtain $A X^{*}=X^{*} A^{*}$ and thus $A H=H A^{*}$ and $A J=J A^{*}$.

Let $\Re$ be the kernel of $H$ and decompose the Hilbert space as $\Re^{\perp} \oplus \Re$. $\Re$ is clearly invariant for $A^{*}$ and we can represent $A$ and $H$ as operator matrices:

$$
A=\left(\begin{array}{ll}
C & D \\
0 & E
\end{array}\right) \text { and } H=\left(\begin{array}{ll}
K & 0 \\
0 & 0
\end{array}\right)
$$

$C$ is $M$-hyponormal and since $A H=H A^{*}$ we have $C K=K C^{*}$ and because $K$ is one-to-one and has dense range we conclude that $C$ is normal by [3, Theorem 3(a)]. By Theorem $\mathrm{A}, D=0$ and it follows that $A^{*} H=H A$. Similarly, $A^{*} J=J A$ and thus $A^{*} X=X A$.

THEOREM 2. If $A$ and $B$ are $M$-hyponormal and $A X=X B^{*}$ then $A^{*} X=X B$.

Proof. Let

$$
\hat{A}=\left(\begin{array}{ll}
A & 0 \\
0 & B
\end{array}\right) \text { and } \hat{X}=\left(\begin{array}{cc}
0 & X \\
0 & 0
\end{array}\right) \text {. }
$$

$\hat{A}$ is $M$-hyponormal and $\hat{A} \hat{X}=\hat{X} \hat{A}^{*}$ and Theorem 1 yields the desired result.

The next theorem, which generalizes Theorem 3(a) of [3], identifies the subspaces on which $A$ and $B$ must be normal.

Theorem 3. Let $A, B$ and $X$ be as in Theorem 2. Then

(a) $(\operatorname{ran} X)^{-}$reduces $A$ and $\operatorname{ker} X$ reduces $B$.

(b) $A \mid(\operatorname{ran} X)^{-}$and $B^{*} \mid \operatorname{ker}^{\perp} X$ are unitarily equivalent normal operators.

Proof. (a) By Theorem 2, $A X X^{*}=X B^{*} X^{*}=X X^{*} A$. Thus $A$ commutes with $X X^{*}$ and so $(\operatorname{ran} X)^{-}=\left(\operatorname{ran} X X^{*}\right)^{-}$reduces $A$. Similarly $B$ commutes with $X^{*} X$ and $\operatorname{ker} X=\operatorname{ker} X^{*} X$ reduces $B$.

(b) Let $X=U P$ be the polar decomposition of $X$. Since $B$ commutes with $P$ as above, we have

$$
\left(A U-U B^{*}\right) P=0 .
$$

Let $\mathscr{K}_{1}=\operatorname{ker}^{\perp} X=\operatorname{ker}^{\perp} P$ and let $\mathscr{K}_{2}=(\operatorname{ran} X)^{-}$; let $A_{2}=A \mid \mathscr{K}_{2}$ and $B_{1}=$ $B \mid \mathscr{K}_{1}$. Let $V: \mathscr{K}_{1} \rightarrow \mathscr{K}_{2}$ be defined by $V f=U f$ for all $f \in \mathcal{K}_{1}$. The equation above then becomes

$$
A_{2} V=V B_{1}^{*} \text {. }
$$

Since $V$ is an invertible isometry we have that $A_{2}$ is unitarily equivalent to $B_{1}^{*}$ and since $A_{2}$ and $B_{1}$ are both $M$-hyponormal, Theorem $B$ implies that both are normal. The proof is complete.

We now proceed to an asymptotic version, which is most readily attained by employing some machinery developed by Berberian [1]. We sketch Berberian's construction here; the details are in [1]. Let $\mathcal{H}$ be a Hilbert space and let $\mathscr{B}$ be the set of bounded sequences of vectors $\left\{f_{n}\right\}$, with $f_{n} \in \mathcal{H}$. Let "glim" denote a generalized limit defined on the collection of bounded sequences of complex numbers, and let $\mathscr{K}=\left\{\left\{f_{n}\right\} \in \mathscr{B}: \operatorname{glim}\left\{\left\|f_{n}\right\|\right\}=0\right\}$. Then the set $\mathscr{P}=\mathscr{B} / \mathscr{K}$ has a pre-Hilbert space structure with the inner product $\left(\left\{f_{n}\right\}+\mathscr{N},\left\{g_{n}\right\}+\mathscr{T}\right)=$ $\operatorname{glim}\left(f_{n}, g_{n}\right)$. The map $f \rightarrow\{f, f, \ldots\}+\mathcal{X}$ is a natural imbedding of $\mathcal{H}$ into $\mathscr{P}$. 
Let $\mathcal{K}$ be the completion of $\mathscr{P}$. If $\left\{T_{n}\right\}$ is a bounded sequence of operators on $\mathcal{H}$ and if $\left\{f_{n}\right\} \in \mathscr{N}$, then the sequence $\left\{T_{n} f_{n}\right\} \in \mathcal{N}$ and it follows that the function that maps $\left\{g_{n}\right\}+\Re$ to $\left\{T_{n} g_{n}\right\}+\Re$ defines a bounded linear operator on $\mathscr{K}$ which we call $\phi\left(\left\{T_{n}\right\}\right)$. It is easy to check that $\phi\left(\left\{T_{n}\right\}\right)=0$ if and only if $\left\|T_{n}\right\| \rightarrow 0$, that $\phi\left(\left\{T_{n}^{*}\right\}\right)=\phi\left(\left\{T_{n}\right\}\right)^{*}$, and that $\phi\left(\left\{T_{n}\right\}\right)$ is positive if and only if $T_{n}-\left|T_{n}\right| \rightarrow 0$, in the strong operator topology.

THEOREM 4. Let $\left\{T_{n}\right\}$ and $\left\{S_{n}\right\}$ be bounded sequences for which there exists a number $M$ such that, for all complex numbers $\lambda$,

$$
\begin{aligned}
& M^{2}\left(T_{n}-\lambda\right)^{*}\left(T_{n}-\lambda\right)-\left(T_{n}-\lambda\right)\left(T_{n}-\lambda\right)^{*} \\
&-\left|M^{2}\left(T_{n}-\lambda\right)^{*}\left(T_{n}-\lambda\right)-\left(T_{n}-\lambda\right)\left(T_{n}-\lambda\right)^{*}\right| \rightarrow 0 \quad \text { (strongly) }
\end{aligned}
$$

and

$$
\begin{aligned}
& M^{2}\left(S_{n}-\lambda\right)^{*}\left(S_{n}-\lambda\right)-\left(S_{n}-\lambda\right)\left(S_{n}-\lambda\right)^{*} \\
& -\left|M^{2}\left(S_{n}-\lambda\right)^{*}\left(S_{n}-\lambda\right)-\left(S_{n}-\lambda\right)\left(S_{n}-\lambda\right)^{*}\right| \rightarrow 0 \quad \text { (strongly). }
\end{aligned}
$$

Let $\left\{X_{n}\right\}$ be a bounded sequence and suppose that $T_{n} X_{n}-X_{n} S_{n}^{*} \rightarrow 0$. Then $T_{n}^{*} X_{n}-X_{n} S_{n} \rightarrow 0$.

Proof. The conditions on $\left\{T_{n}\right\}$ and $\left\{S_{n}\right\}$ imply that $\phi\left(\left\{T_{n}\right\}\right)$ and $\phi\left(\left\{S_{n}\right\}\right)$ are $M$-hyponormal. The equation

$$
\phi\left(\left\{T_{n}\right\}\right) \phi\left(\left\{X_{n}\right\}\right)=\phi\left(\left\{X_{n}\right\}\right) \phi\left(\left\{S_{n}\right\}\right)^{*}
$$

holds, and Theorem 2 yields the result.

CoRollaRY. If $T$ and $S$ are $M$-hyponormal and if $T X_{n}-X_{n} S^{*} \rightarrow 0$ for a bounded sequence $\left\{X_{n}\right\}$, then $T^{*} X_{n}-X_{n} S \rightarrow 0$ as well.

\section{REFERENCES}

1. S. K. Berberian, Approximate proper vectors, Proc. Amer. Math. Soc. 13 (1962), 111-114.

2. 113-114.

3. M. Radjabalipour, Majorization and normality of operators, Proc. Amer. Math. Soc. 62 (1977), 105-110.

4. J. G. Stampfli and B. L. Wadhwa, On dominant operators, Monatsh. Math. 84 (1977), 143-153.

Department of Mathematics, University of Alabama, University, Alabama 35486

Department of Mathematics, Texas A\&M Untversity, College Station, Texas 77843

Department of Mathematics, Universtty of Alabama, University, Alabama 35486 